\title{
Mechanical Properties and Failure Analysis of Visible Light Crosslinked Alginate-Based Tissue Sealants
}

Patrick Charron ${ }^{1 \dagger}$, Spencer L. Fenn ${ }^{2 \dagger}$, Alex Poniz ${ }^{1}$, Rachael A. Oldinski ${ }^{1,2,3 *}$

${ }^{1}$ Mechanical Engineering Program, College of Engineering and Mathematical Sciences, Univeristy of Vermont;

${ }^{2}$ Bioengineering Program, School of Engineering and College of Medicine, University of Vermont;

${ }^{3}$ Department of Medicine, College of Medicine, University of Vermont, Burlington, VT

$\dagger$ Equal Authorship

*Contact/Corresponding Author:

Rachael A. Oldinski

33 Colchester Ave

Burlington, VT 05405

Oldinski@uvm.edu

802-656-3338

Keywords: Visible light crosslinking; alginate; oxidation; burst pressure; tissue sealant

\begin{abstract}
Moderate to weak mechanical properties limit the use of naturally-derived tissue sealants for dynamic medical applications, e.g., sealing a lung leak. To overcome these limitations, we developed visible-light crosslinked alginate-based hydrogels, as either non-adhesive methacrylated alginate (Alg-MA) hydrogel controls, or oxidized Alg-MA (Alg-MA-Ox) tissue adhesive tissue sealants, which form covalent bonds with extracellular matrix (ECM) proteins. Our study investigated the potential for visible-light crosslinked Alg-MA-Ox hydrogels to serve as effective surgical tissue sealants for dynamic in vivo systems. The Alg-MA-Ox hydrogels were designed to be an injectable system, curable in situ. Burst pressure experiments were conducted on a custom-fabricated burst pressure device using constant air flow; burst pressure
\end{abstract}


properties and adhesion characteristics correlated with the degrees of methacrylation and oxidation. In summary, visible light crosslinked Alg-MA-Ox hydrogel tissue sealants form effective seals over critically-sized defects, and maintain pressures up to $50 \mathrm{~mm} \mathrm{Hg}$.

\section{Introduction}

For several decades, breakthroughs in medical technologies have improved patient care while also reducing the invasiveness and recovery time for many procedures. In particular, advances in laparoscopic surgery have allowed for internal procedures to be performed through small incisions, decreasing the need for many open-body operations.[1-3] In these minimally-invasive surgeries, common approaches to rejoin or seal tissues, such as sutures or staples, can be difficult to implement, may cause further damage to tissue, and often fail to completely seal tissue resulting in the leakage of bodily fluids or air.[1, 4] An alternative method for providing a simple, immediate seal for wound closure is the use of new engineered tissue sealants, which are applied as a liquid and activated on demand to adhere, cure, and bond in situ. [1,5]

Tissue sealants are commonly formed using natural or synthetic polymers, or both. Synthetic sealants, capable of higher mechanical strengths and tissue-bonding properties, are potentially cytotoxic, may cause chronic inflammation, and exhibit low adherence to wet tissues. [4] Natural based sealants are advantageous compared to their synthetic counterparts due to heightened biocompatibility, degradability, sustainable derivation, and intrinsic bioactive qualities.[6-11] The abundant resource, propensity for chemical modification, and enhanced biocompatibility, degradability, and non-toxicity make alginate and alginate-based hydrogels prime candidates for tissue sealant applications.[7, 8, 12] Alginate, derived from brown algae, is desirable not only for those attributes already listed, but also for its relatively low cost and historical use in drug delivery and tissue engineering. $[7,8]$ While alginate hydrogels can form films in situ via calcium crosslinking, these materials are relatively weak (i.e., low elasticity, toughness), and exhibit relatively quick degradation times.

Chemical modification of alginate and similar polysaccharides allows for designed physical and mechanical properties of engineered alginate hydrogels through controlled covalent crosslinking, thus addressing limitations associated with ionically crosslinked alginate hydrogels.[7, 11, 13, 
14] The methacrylation of alginate imparts an acrylate functional group capable of covalent crosslinking with itself by free radical polymerization in the presence of a photoinitiator.[7, 14, 15] While tradition photo-crosslinking mechanisms often employ ultraviolet (UV) light activation, UV exposure is cytotoxic and degrades biological molecules (e.g., drugs).[14, 16-20] As a safer alternative, methacrylated alginate (Alg-MA) also covalently crosslinks upon exposure to visible light in the presence of the photosensitizer, Eosin Y.[12, 15, 21-23] Furthermore, while alginate is inherently non-adhesive, oxidation of the backbone structure [24, 25] will elicit functional aldehyde groups capable of forming covalent bonds with amine groups present on tissue (i.e., imine bond formation) like those found in extracellular matrix (ECM) proteins.[26-28]

Burst pressure testing is a useful means of analysis for tissue sealants, as it allows for quantification of the sealant mechanical strength under characteristic loading conditions. Burst pressures were collected following a modified standard (ASTM F2392), which quantifies not only maximum burst pressures, but also the mode of failure (cohesive, i.e., material failure, versus adhesive failure) for the various hydrogel compositions.[29] In addition, a video recording system may be employed to further characterize the mode of sealant failure. Burst pressure and failure data analysis provides a direct comparison of tissue sealant mechanical behavior and also enables indirect insight into the effects of material integrity and tissue adhesion; burst pressure experiments and rheological characterization of novel hydrogels provides insight into material structure-function relationships, in order to optimize material properties for biomedical applications.

This paper presents the fabrication and characterization of Alg-MA-Ox tissue sealants, specifically for use in sealing damaged lung tissue. Our investigation seeks to quantitatively assess the effect of modulating the degree of methacrylation and oxidation on adhesion and burst pressure properties. The novelty of our approach lies in the implementation of both methacrylation and oxidation chemistries to vary mechanics and adhesion, as well as our use of visible light crosslinking to reduce the cytotoxic effects observed with UV-cured sealants. We hypothesized that an Alg-MA-Ox hydrogel will form an effective seal over an air leak upon exposure to visible light. Furthermore, we hypothesized that the Alg-MA-Ox hydrogels would 
adhere to an ECM substrate and exhibit high burst pressures in a physiologically-relevant environment. To test these hypotheses, a custom burst pressure device was designed, fabricated, and implemented for the quantitative and repeatable mechanical analysis of visible-light crosslinked alginate-based hydrogel tissue sealants.

\section{Materials and Methods}

\subsection{Synthesis of methacrylated alginate (Alg-MA)}

Alg-MA was synthesized using an aqueous-based reaction. One percent (w/v) sodium alginate (Manugel, Mw $\approx 200 \mathrm{kDa}$, FMC Biopolymer) solution, in deionized (DI) water, was reacted with a 20-molar excess of methacrylic anhydride (Sigma Aldrich) for 12 hours.[12, 15, 30-32] The reaction product was treated with sodium hydroxide and dialyzed in DI water for 3 days. The degree of methacrylation (DOM), or ratio of methacrylate groups per repeat unit of alginate, was determined using ${ }^{1} \mathrm{H}-\mathrm{NMR}$ spectroscopy (Bruker AVANCE III $500 \mathrm{MHz}$ high-field NMR spectrometer). Briefly, Alg-MA was dissolved in deuterium oxide $\left(\mathrm{D}_{2} \mathrm{O}\right)$ at $1 \%(\mathrm{w} / \mathrm{v})$ solution and was analyzed at room temperature, spinning at $20 \mathrm{~Hz}$ for 16 scans. The DOM was determined by calculating ratios of methylene $(6.1,5.6 \mathrm{ppm})$ and alginate methyl (1.9 ppm) peak integrations.[15] Alg-MA material with a single DOM was used for subsequent rheological and mechanical analysis, and modification via oxidation.

\subsection{Synthesis of oxidized methacrylated alginate (Alg-MA-Ox)}

Alg-MA was reacted with sodium periodate (Sigma) to open the uronate residues, which form the alginate backbone, creating an aldehyde group.[26, 33] One percent (w/v) alginate solutions were prepared by dissolving sodium alginate or Alg-MA in DI water. Different volumes of a 3\% $(\mathrm{w} / \mathrm{v})$ sodium periodate solution were added to the alginate solutions and reacted in the dark for 24 hours at room temperature to achieve distinctly different degrees of oxidation (DOO). The Alg-MA and sodium periodate solutions were mixed together and stirred for 24 hours in a dark environment at room temperature. The product, Alg-MA-Ox, was purified by dialysis in DI water (MWCO $6 \mathrm{kDa}$, Thermo-Fisher) for three days and lyophilized. Theoretical DOO values were 10, 30 and 50\% modification; experimental values were determined by comparing the ratio of alginate methyl protons (5.5 and $5.0 \mathrm{ppm}$ ), to the newly formed methyl protons (5.0 ppm).[26] In addition, the DOM was reassessed after the oxidation reaction (see methods above). 


\subsection{Polymer characterization and visible light crosslinking}

Viscosity and shear stress, as functions of shear rate, were evaluated on Alg-MA and Alg-MAOx hydrogel sealant precursor solutions (i.e., prior to photo-crosslinking). All measurements were carried out on a rheometer (AR2000, TA Instruments) equipped with a Peltier plate maintained at $25^{\circ} \mathrm{C}$, using a $40 \mathrm{~mm}$ diameter $1^{\circ} 59^{\prime} 47^{\prime \prime}$ hard anodized aluminum cone geometry. Alginate and chemically-modified alginate powders were added to DI water to form 3\% (w/v) alginate solutions of the following compositions: alginate controls, Alg-MA (DOM = 77), AlgMA-Ox $(\mathrm{DOO}=10,30,50)$, and 50:50 polymer blends of Alg-MA and Alg-MA-Ox materials. The control and modified alginate solutions were blended with photo-activators to enable photocrosslinking: $1 \mathrm{mM}$ Eosin Y (photo-sensitizer), $125 \mathrm{mM}$ triethanolamine (photo-initiator), 20 $\mathrm{mM}$ 1-vinyl-2-pyrrolidinone (catalyst).[15, 21,22] Aliquots of $580 \mu \mathrm{L}$ were placed within a 27 $\mu \mathrm{m}$ gap between the Peltier plate and cone geometry. Shear sweeps were performed to determine the viscosity (Pa.s) and shear stress $(\mathrm{Pa})$ of the alginate-based solutions at 1\% radial strain, 1-100 $(1 / s)$, over one min $(n=4)$. Data was analyzed using analytical software (TA Data Analysis); data sets for various samples within a single group are merged and sorted by shear rate, and plotted on the same graph.

Oscillatory time sweeps at $10 \%$ radial strain and $1 \mathrm{~Hz}$ were conducted on alginate-based hydrogel precursor solution during exposure to green light $(525 \mathrm{~nm}$, custom $9.84 \mathrm{~cm}$ diameter LED ring, NFLS-G30X3-WHT, SuperBrightLEDs) over a period of 10 minutes (600 seconds).[14] Data collection started upon the start of light exposure (i.e., photo-crosslinking activation). Shear storage $\left(\mathrm{G}^{\prime}\right)$ and loss $\left(\mathrm{G}^{\prime \prime}\right)$ moduli were calculated, and tan delta (ratio of $\mathrm{G}^{\prime \prime}$ to $\mathrm{G}^{\prime}$ ) was analyzed using analytical software (TA Data Analysis).

\subsection{Burst pressure testing and failure analysis}

Burst pressure values were determined using ASTM F2392 as a guide.[29] Burst pressure was recorded using a custom-designed pressure-chamber testing device according to the ASTM standard (Figure 1). The main chamber was machined from polyether ether ketone (PEEK) to provide low cost fabrication and fluid-related degradation resistance; it was designed to be two sections fastened by two bolts with a fluoroelastomer o-ring to provide an airtight seal, for easy 
specimen loading between the sections. A syringe pump (Harvard Apparatus PHD 2000 Infusion) controlled the fluid injection rate into the chamber; for this experiment we used air pressure. A pressure transducer (Omega PX-409-030AUSBH) was connected to the pressure chamber through a NPT port, allowing for accurate, real-time data acquisition via a connected computer (Figure 1).

Collagen substrates (collagen casing, The Sausage Maker Inc.) were hydrated in DI water and tested on the pressure device before creating a defect (i.e., puncture) to ensure that the substrate was intact and void of defects, and formed a leak-free seal. The substrates were punctured with a $3 \mathrm{~mm}$ diameter biopsy punch and re-tested to ensure a through-thickness defect was formed resulting in a drop in pressure (or the inability to hold pressure). Punctured collagen substrates were clamped between a glass slide and a Teflon mold, with a $1.5 \mathrm{~cm}$ diameter hole, centered over the collagen puncture. Hydrogel precursor solution $(0.5 \mathrm{~mL})$ was deposited into the mold, over the collagen puncture, and photo-crosslinked for 10 minutes under visible green light (523 $\mathrm{nm})$. The collagen substrate with the hydrogel sealant was removed from the mold and loaded onto the burst pressure device. Air volume, via the syringe pump, was increased at a constant air flow rate into the pressure chamber, until the seal failed; failure was defined as a loss in pressure due to a leak in seal (i.e., experimental sample). All burst pressure tests were conducted at room temperature with compressed air at an infusion rate of $75 \mathrm{~mL} / \mathrm{hr}$.

The hydrogel sealant failure mechanism, either hydrogel delamination from the collagen substrate or hydrogel material failure (i.e., failure of the material under loading), was assessed via visual inspection of the hydrogel materials and a digital microscope (Dino-Lite Pro AM413TA, Dino-Lite Digital Microscopes) during and after testing. Video was collected directly above the burst pressure device for the duration of the test. The digital microscope was positioned 10-15 $\mathrm{cm}$ above the hydrogel sealant, and recording was initiated just prior to the activation of the syringe pump and halted several seconds after sealant failure (supplemental videos have been edited to show failure activity). Delamination, i.e., adhesive failure, was classified as bond failure between the sealant and substrate, whereas material failure was classified as bond failure within the material itself. 


\section{Results and Discussion}

\subsection{Rheology}

The chemical modification of alginate, and other polysaccharides, may induce chain scission and a reduction in molecular weight of the alginate backbone; polymer degradation depends on the nature of the chemical reaction.[20, 26, 27, 33] Also, sequential chemical reactions will affect the degree of modifications of various functional groups. Therefore, DOO and DOM were calculated for each modified alginate formulation. All of the Alg-MA starting materials had a $\mathrm{DOM}=77$; however, as evidenced by the DOM calculations performed on the Alg-MA-Ox materials, the oxidation reaction slightly reduced the DOM, as shown in Table 1.

Physical changes, such as a reduction in molecular weight, result in variable mechanical responses. Thus, we quantified viscosity and shear stress after each chemical reaction; the effects of shear rate on the viscosity and shear stress of $3 \%(\mathrm{w} / \mathrm{v})$ alginate solutions are shown in Figures 2A and 2B, respectively. Solution viscosities decreased exponentially with increasing shear rates for all solutions tested. Compared to non-modified alginate solutions (i.e., controls), the methacrylated alginate (Alg-MA) solution maintained high viscosities (Figure 2A) and shear stresses (Figure 2B). In contrast, the oxidized and methacrylated alginate (Alg-MA-Ox) solutions demonstrated much lower viscosities and shear stresses when compared to alginate and Alg-MA solutions. Methacrylation chemistry is deleterious to the alginate backbone, although this is more pronounced in Alg-MA-Ox materials.[26, 27, 33] During the oxidation reaction, alginate molecular weight was reduced, and resulted in a $>100$-fold reduction in viscosity. During the oxidation reaction, Alg-MA was exposed to high concentrations of sodium periodate to open the uronate residues on the acidic repeat units, rendering the polymer reactive with ECM proteins (i.e., imine formation) through the introduction of aldehyde functional groups.[20, 34] Additionally, the oxidation of alginate increases susceptibility to hydrolytic degradation, improving biodegradability of the sealant.[33] Thus, tissue adhesion and in vivo degradation time can be controlled through the optimization of the addition and creation of acrylate and aldehyde functionalities to alginate. Adhesion may be further assisted through the formation of weak hydrogen bonds between the biomacromolecules comprising the sealant and the proteins present on the tissue surface.[35] 
To effectively seal a leak or damaged tissue, a liquid tissue sealant must display a relatively high viscosity to maintain a film over a tissue defect during the gelation, or curing, process. While the Alg-MA-Ox material is required to maintain adhesion to underlying tissue, the requirements of the amount and/or degree of oxidation have not been studied. Thus, Alg-MA was blended with Alg-MA-Ox materials to enhance the viscosity of the solutions and further improve material properties. Alg-MA and Alg-MA-Ox blends (1:1) were created from the same formulation of Alg-MA, and different Alg-MA-Ox polymers with varying degrees of oxidation (10, 30 and 50\%). Upon blending Alg-MA with Alg-MA-Ox, viscosity and shear stress values were partially recovered compared to Alg-MA-Ox solutions. The viscosity and shear stress values for all of the polymer blend solutions were similar, suggesting that the properties of the Alg-MA polymer were dominant and served as an equalizing agent (see Figure 2).

\subsection{Visible light crosslinking and gelation time}

Alg-MA and Alg-MA-Ox solutions, in the presence of photo-activators, were crosslinked and formed into hydrogels upon exposure to green light. The gelation times of the hydrogel solutions were determined from oscillatory time sweep plots (Figure 3A, C). The initiation of gelation, i.e., gelation time, was determined to be at the inflection point of the delta versus time curve. The Alg-MA solution and Alg-MA-Ox polymer blend solutions indicate a clear inflection in the delta curve, indicative of successful photo-initiation and subsequent crosslinking. The Alg-MA-Ox solutions with $\mathrm{DOO} \geq 30$ failed to form a gel (i.e., photo-crosslink) rheometry. The storage moduli curves versus time are a more reliable indicator for terminal crosslinking as it plateaus and indicates no further crosslinking (Figure 3B, D). Upon inspection of the $\mathrm{G}^{\prime}$ curves, a majority of the crosslinking was completed by 600 seconds, and increased by several orders of magnitude. Thus, an exposure time of 10 minutes (600 seconds) was used to form hydrogels for burst testing.

\subsection{Burst pressure properties}

To perform effectively, a tissue sealant must adhere to underlying tissue and exhibit material strength exceeding physiological loading conditions. Burst pressure measurements and the subsequent failure analysis quantified the ability for alginate-based hydrogels to perform as tissue sealants under increasing pressure. Two modes of failure, delamination of the material 
from the substrate, and material rupture due to a loss in strength, were determined via video during experimentation (Figure 4A). Representative pressure versus time curves from burst pressure testing are shown in Figure 4B.

While the Alg-MA hydrogel saw the highest individual burst pressure, it was also the only material to fail by delamination. Burst volume measurements were calculated based on the amount of air pumped into the device before failure. The burst pressure volumes relate to the qualitative flexibility of the tissue sealant (Figure 4C). Comparing the 10 Alg-MA-Ox and 10 Alg-MA-Ox blend sample groups, the flexibility of the blend material resulted in a higher burst pressure and burst volume.

As the DOM decreases, the number of methacrylate functional groups available for covalent crosslinking decreases. With less methacrylate groups available, the resulting polymerized hydrogel is more loosely bound together than its highly methacrylated counterpart. Polymer networks that are more loosely bound behave more as a viscous material rather than an elastic material, and exhibit lower storage moduli, as shown in Table 1 (see above). Because our polymer hydrogels rely more on elastic than viscous behavior, post-crosslinking, for its application as a sealant, it is expected that storage modulus would correlate with burst pressure. The relation between DOM, storage modulus, and burst pressure are highlighted in Table $\mathbf{1}$.

Delamination, or adhesive failure, occurred when the hydrogel patch separated from the substrate with no visible sign of material damage. Material failure was characterized as the failure of the sealant material immediately above the puncture, and showing visible damage to the material (Supplemental videos 1 and 2). The Alg-MA hydrogel sealants failed exclusively through delamination, while the Alg-MA-Ox materials exhibited material failure, with the exception of a single 10 DOO Alg-MA-Ox:Alg-MA blend experimental group. Alginate is inherently nonadhesive, and there are few forces keeping the Alg-MA hydrogel sealant adhered to the collagen substrate. However, the Alg-MA-Ox hydrogels and blends interact with the collagen substrate through functional aldehyde groups, preventing delamination before material failure. The only case of delamination outside the Alg-MA group occurred in the blend with the lowest theoretical DOO, resulting in fewer aldehyde groups to interact with the substrate, possibly explaining the 
delamination. In summary, aldehyde functionality is required to ensure adhesion and the formation of a seal. However, the optimum DOO required for maintaining tissue adhesion while exhibiting high burst pressures has yet to be determined.

\section{Conclusion}

Alginate, a biomacromolecule derived from brown algae, was investigated as a hydrogel tissue sealant. Chemical modifications were tuned to optimize the mechanical and adhesive properties, which are essential in developing a tissue sealant. Specifically, methacrylation resulted in acrylate groups that were photo-responsive in the presence of photo-activators and visible green light. Alginate oxidation created aldehyde groups for sealant-substrate interactions and increased adhesion to tissue matrix proteins. A custom-designed burst pressure device was fabricated and implemented in the mechanical analysis of alginate-based hydrogel sealants. While Alg-MA hydrogels were able to withstand high pressures without mechanically failing, these materials delaminated from the substrate and failed adhesively. Conversely, Alg-MA-Ox formed hydrogels that adhered to the substrate; however, these materials failed at lower burst pressures. Alg-MA and Alg-MA-Ox polymer blends benefited from the high mechanical strength of the Alg-MA and the adhesiveness of the Alg-MA-Ox. Most notably, higher DOO Alg-MA-Ox did not result in improved adhesion, which may also be the result of polymer degradation during the oxidation reaction. Indeed, minimal oxidative degradation (1-5\% DOO) is required to induce hydrogel sealant adhesion while maintaining hydrogel network integrity.

\section{Acknowledgements}

This work was funded in part by NIH Grant T32 HL076122 (SL Fenn training fellowship) as well as the University of Vermont College of Engineering and Mathematical Sciences.

\section{References}

[1] Annabi N, Tamayol A, Shin SR, Ghaemmaghami AM, Peppas NA, Khademhosseini A. Surgical materials: Current challenges and nano-enabled solutions. Nano today 2014;9:574-89. [2] Rané A, Rao P, Rao P. Single-port-access nephrectomy and other laparoscopic urologic procedures using a novel laparoscopic port (R-port). Urology 2008;72:260-3.

[3] Streuli CH, Bailey N, Bissell MJ. Control of mammary epithelial differentiation: basement membrane induces tissue-specific gene expression in the absence of cell-cell interaction and morphological polarity. The Journal of Cell Biology 1991;115:1383-95. 
[4] Annabi N, Yue K, Tamayol A, Khademhosseini A. Elastic sealants for surgical applications. European Journal of Pharmaceutics and Biopharmaceutics 2015.

[5] Oliveira CLd, Santos CHMd, Bezerra FMM, Bezerra MM, Rodrigues LdL. Utilização de adesivos de cianoacrilatos em suturas de pele. Rev bras cir plást 2010;25:573-6.

[6] Draget KI, Taylor C. Chemical, physical and biological properties of alginates and their biomedical implications. Food Hydrocolloids 2011;25:251-6.

[7] Lee KY, Mooney DJ. Alginate: properties and biomedical applications. Progress in polymer science 2012;37:106-26.

[8] Lee KY, Mooney DJ. Hydrogels for tissue engineering. Chemical reviews 2001;101:1869-80.

[9] Place ES, Evans ND, Stevens MM. Complexity in biomaterials for tissue engineering. Nature materials 2009;8:457-70.

[10] Langer R. Biodegradable polymer scaffolds for tissue engineering. Nat Biotechnol 1994.

[11] Moller L, Krause A, Dahlmann J, Gruh I, Kirschning A, Drager G. Preparation and evaluation of hydrogel-composites from methacrylated hyaluronic acid, alginate, and gelatin for tissue engineering. International Journal of Artificial Organs 2011;34:93-102.

[12] Wagner DE, Fenn SL, Bonenfant NR, Marks ER, Borg Z, Saunders P, et al. Design and Synthesis of an Artificial Pulmonary Pleura for High Throughput Studies in Acellular Human Lungs. Cell Mol Bioeng 2014;7:184-95.

[13] Zhao X, Huebsch N, Mooney DJ, Suo Z. Stress-relaxation behavior in gels with ionic and covalent crosslinks. Journal of Applied Physics 2010;107:063509-5.

[14] Fenn SL, Oldinski RA. Visible light crosslinking of methacrylated hyaluronan hydrogels for injectable tissue repair. J Biomed Mater Res B Appl Biomater 2015.

[15] Smeds KA, Grinstaff MW. Photocrosslinkable polysaccharides for in situ hydrogel

formation. Journal of biomedical materials research 2001;54:115-21.

[16] Kulms D, Schwarz T. Molecular mechanisms involved in UV-induced apoptotic cell death.

Skin Pharmacology and Physiology 2002;15:342-7.

[17] Godar DE. Preprogrammed and Programmed Cell Death Mechanisms of Apoptosis:

UV-Induced Immediate and Delayed Apoptosis. Photochemistry and photobiology 1996;63:82530.

[18] Bendjennat M, Boulaire J, Jascur T, Brickner H, Barbier V, Sarasin A, et al. UV irradiation triggers ubiquitin-dependent degradation of $\mathrm{p} 21 \mathrm{WAF} 1$ to promote DNA repair. Cell 2003;114:599-610.

[19] Guieysse B, Viklund G, Toes A-C, Mattiasson B. Combined UV-biological degradation of PAHs. Chemosphere 2004;55:1493-9.

[20] Gomez CG, Rinaudo M, Villar MA. Oxidation of sodium alginate and characterization of the oxidized derivatives. Carbohydrate Polymers 2007;67:296-304.

[21] Nettles DL, Vail TP, Morgan MT, Grinstaff MW, Setton LA. Photocrosslinkable hyaluronan as a scaffold for articular cartilage repair. Annals of biomedical engineering 2004;32:391-7.

[22] Park YD, Tirelli N, Hubbell JA. Photopolymerized hyaluronic acid-based hydrogels and interpenetrating networks. Biomaterials 2003;24:893-900.

[23] Wagner DE, Fenn SL, Bonenfant NR, Marks ER, Borg Z, Saunders P, et al. Design and synthesis of an artificial pulmonary pleura for high throughput studies in acellular human lungs. Cellular and molecular bioengineering 2014;7:184-95. 
[24] Gao C, Liu M, Chen S, Jin S, Chen J. Preparation of oxidized sodium alginate-graft-poly((2dimethylamino) ethyl methacrylate) gel beads and in vitro controlled release behavior of BSA. Int J Pharm 2009;371:16-24.

[25] Balakrishnan B, Lesieur S, Labarre D, Jayakrishnan A. Periodate oxidation of sodium alginate in water and in ethanol-water mixture: a comparative study. Carbohydrate research 2005;340:1425-9.

[26] Jeon O, Alt DS, Ahmed SM, Alsberg E. The effect of oxidation on the degradation of photocrosslinkable alginate hydrogels. Biomaterials 2012;33:3503-14.

[27] Jeon O, Samorezov JE, Alsberg E. Single and dual crosslinked oxidized methacrylated alginate/PEG hydrogels for bioadhesive applications. Acta Biomater 2014;10:47-55.

[28] Bouten PJM, Zonjee M, Bender J, Yauw STK, van Goor H, van Hest JCM, et al. The chemistry of tissue adhesive materials. Progress in Polymer Science 2014;39:1375-405.

[29] ASTM. Standard Test Method for Burst Strength of Surgical Sealants. West Conshohocken, PA: ASTM International; 2004.

[30] Oudshoorn MHM, Rissmann R, Bouwstra JA, Hennink WE. Synthesis of methacrylated hyaluronic acid with tailored degree of substitution. Polymer 2007;48:1915-20.

[31] Erickson IE, Huang AH, Sengupta S, Kestle S, Burdick JA, Mauck RL. Macromer density influences mesenchymal stem cell chondrogenesis and maturation in photocrosslinked hyaluronic acid hydrogels. Osteoarthritis Cartilage 2009;17:1639-48.

[32] Burdick JA, Chung C, Jia X, Randolph MA, Langer R. Controlled degradation and mechanical behavior of photopolymerized hyaluronic acid networks. Biomacromolecules 2005;6:386-91.

[33] Bouhadir KH, Lee KY, Alsberg E, Damm KL, Anderson KW, Mooney DJ. Degradation of Partially Oxidized Alginate and Its Potential Application for Tissue Engineering. Biotechnology Progress 2001;17:945-50.

[34] Fortune DH, Kettlewell G, Mandley DJ, Thompson I, Cook D. Tissue-adhesive formulations. US7727547 B2. U.S.June 2010.

[35] Berg JM TJ, Stryer L. Section 1.3, Chemical Bonds in Biochemistry. Biochemistry. 5th Edition ed. New York: W H Freeman; 2002.

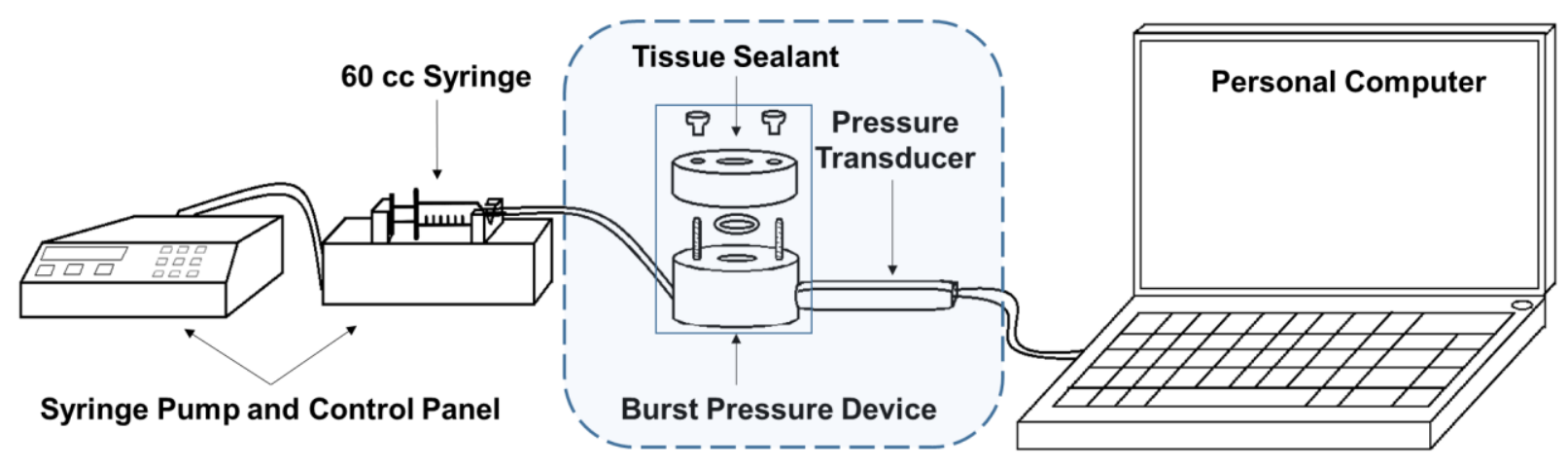

Figure 1. Schematic illustration of experiment setup for mechanical burst pressure testing of alginate-based sealants. 

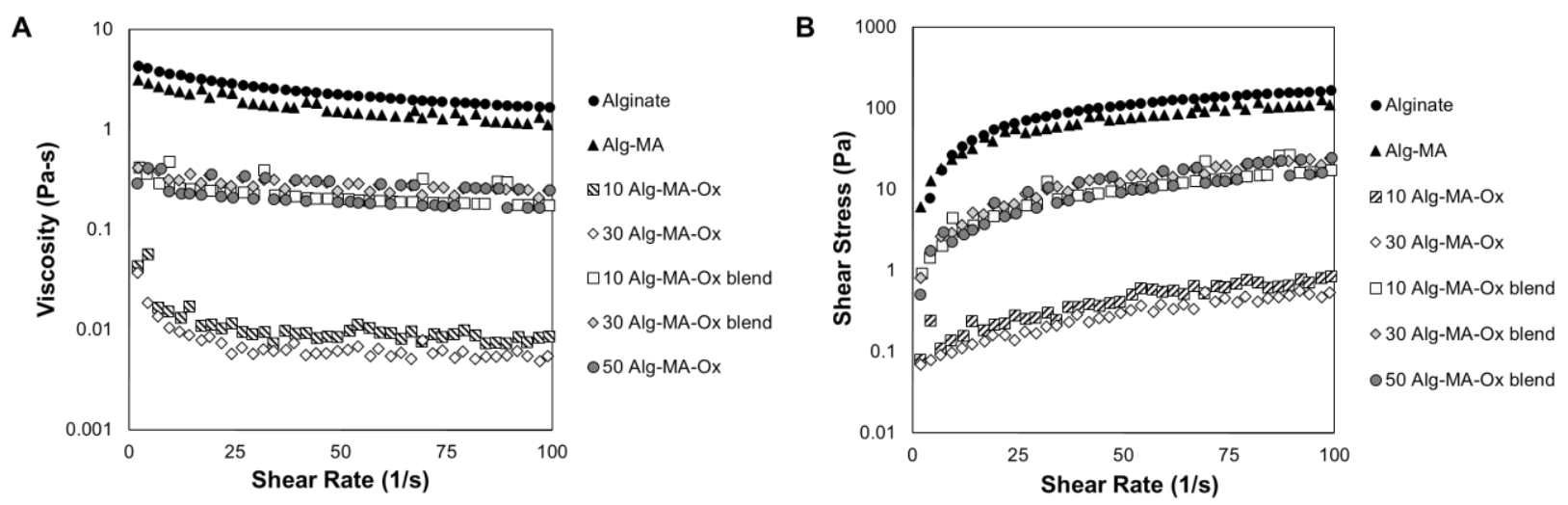

Figure 2. (A) Viscosity (Pa.s) and (B) shear stress (Pa) values were collected for alginate-based tissue sealant precursor solutions, including: Alg-MA, oxidized Alg-MA, and homogenous 50:50 blends of Alg-MA and Alg-MA-Ox. Representative plots for each control and experimental group are shown as average values $(n=4)$.
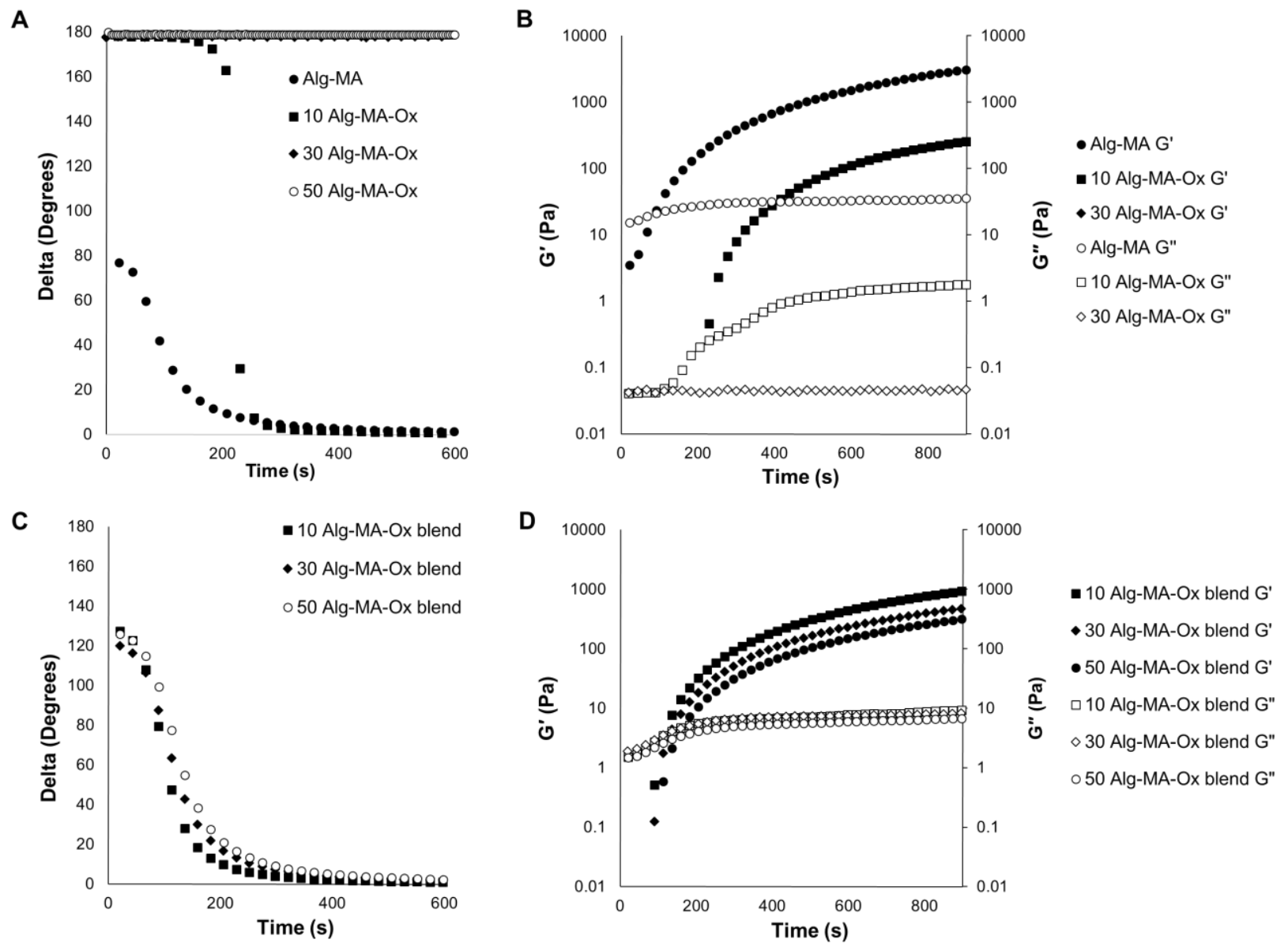

Figure 3. Gelation of alginate-based sealants were assessed using oscillatory time sweeps at $10 \%$ radial strain and $1 \mathrm{~Hz}$ during exposure to green light $(525 \mathrm{~nm})$ over a period of 10 minutes. (A, 
B) Alg-MA and oxidized Alg-MA, and (C, D) homogenous 50:50 blends of Alg-MA and AlgMA-Ox. Delta values decreased as crosslinking occurred via visible light exposure to form hydrogels (A, C). Storage moduli values, G', and loss moduli values, G", were collected during gelation (B, D). Representative plots for each control and experimental group are shown as average values $(n=4)$.

A

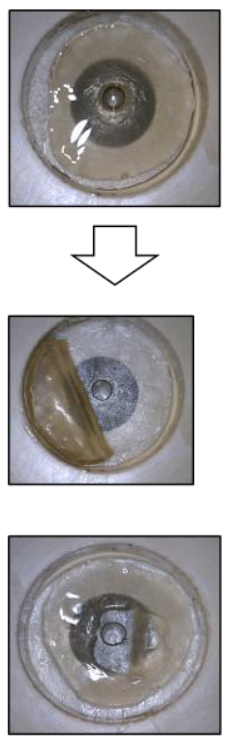

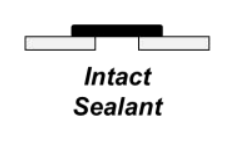
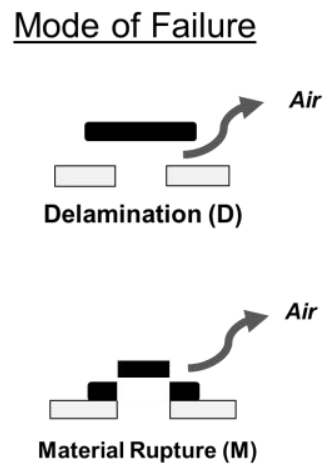

B

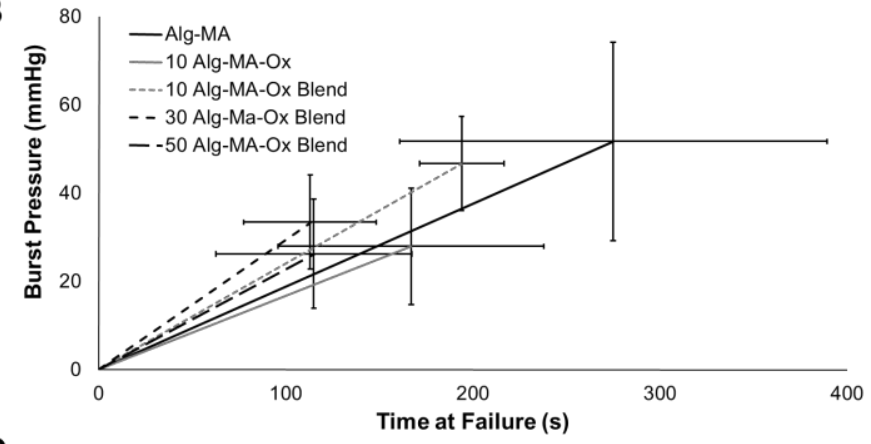

C

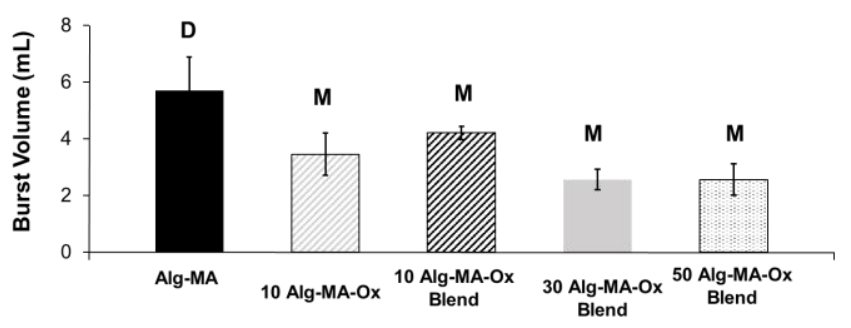

Figure 4. Failure analysis data of alginate-based tissue sealants using a burst pressure device and test system. (A) High-speed video captured the qualitative mode of failure for a sealed $3 \mathrm{~mm}$ diameter hole. The two modes of failure observed were delamination, $\mathrm{D}$, and material failure, $\mathrm{M}$. (B) Burst pressure data is shown as strain to failure with time. While the pressure input rate was constant, materials responded according to their elasticity. Burst pressure was recorded as the highest pressure achieved. Vertical error bars represent burst pressure standard deviation; horizontal error bars represent time to failure $(n=4)$. (C) Total air volume retained in the device before failure was calculated; averages including standard deviations are reported $(n=4)$.

Table 1. Summary of the chemical characterization, burst pressure values, and mode of failure for Alg-MA, Alg-MA-Ox, and homogenous 50:50 polymer blends of Alg-MA and Alg-MA-Ox hydrogel sealants. 


\begin{tabular}{|c|c|c|c|c|c|}
\hline Group & DOM (\%) & DOO (\%) & $\begin{array}{c}\mathbf{G}^{\prime}(\mathbf{P a}) \\
\text { at } \mathbf{t}=\mathbf{6 0 0} \mathbf{~ s}\end{array}$ & $\begin{array}{c}\text { Burst Pressure } \\
(\mathbf{m m H g})\end{array}$ & $\begin{array}{c}\text { Principal } \\
\text { Mode of Failure }\end{array}$ \\
\hline Alg-MA & 77 & 0 & $\sim 1560$ & $49.41 \pm 22.46$ & $\mathrm{D}$ \\
\hline 10 Alg-MA-Ox & 70 & 10 & $\sim 113$ & $26.11 \pm 13.13$ & $\mathrm{M}$ \\
\hline 30 Alg-MA-Ox & 64 & 25 & $<0.01 \mathrm{~Pa}$ & $7.09 \pm 9.85$ & $\mathrm{M}$ \\
\hline 10 Alg-MA-Ox Blend & 74 & 5 & $\sim 440$ & $48.04 \pm 10.64$ & $\mathrm{M}$ \\
\hline 30 Alg-MA-Ox Blend & 71 & 13 & $\sim 235$ & $35.40 \pm 10.66$ & $\mathrm{M}$ \\
\hline 50 Alg-MA-Ox Blend & 54 & 20 & $\sim 150$ & $27.66 \pm 12.36$ & $\mathrm{M}$ \\
\hline
\end{tabular}

